\title{
VII. Uxor, Mater, Socia
}

"Vater ist wieder für einige Zeit in Genf“, schrieb Karin Schauff am Neujahrstag 1958 an ihren Sohn Markus, der sich in einem Schweizer Internat befand und sich zu diesem Zeitpunkt in den Ferien auf der Farm in Brasilien aufhielt, „nachdem er nach Deiner Ablieferung am Flugplatz in Rio zunächst noch einige weitere Reisen in beruflichen Angelegenheiten durch Südamerika unternahm."1 Johannes Schauff verbrachte zweimal im Jahr seinen Urlaub auf der eigenen Fazenda; sie wurde ansonsten weitgehend von seiner Frau betreut, die zwischen Südtirol und Brasilien hin und her pendeln mußte.

Die Tätigkeit Schauffs für die ICMC währte fast zehn Jahre; sieben brachte er fast ausschließlich in Genf zu. Dies ließ ihm wenig Zeit, sich um seine Kinder zu kümmern, die sich alle in schulischer und beruflicher Ausbildung befanden. Gleichwohl erfüllte es den Vater mit Stolz, daß die fünf Söhne trotz des Studiums in Europa alle direkt oder mittelbar in der Landwirtschaft tätig wurden, davon vier in Brasilien. Seinen Sohn Tobias, der in die Verwaltung des elterlichen Anwesens eintrat, wies er geduldig und in zahlreichen Briefen in seine neue Aufgabe $e^{2}{ }^{2}$.

Während der beruflichen Abwesenheit Schauffs kümmerte sich Karin Schauff nicht nur um die materiellen Dinge - es galt vor allem, die große Familie zu versorgen und ihre Ausbildung sicherzustellen. Die örtliche Basis für all diese Unternehmungen war das Anwesen in Löwenegg, mit dem die Familie Schauff durch den "Freund Ludwig Kaas" seit ihrer Emigration nach Italien aufs engste verbunden war ${ }^{3}$. Wie schon erwähnt, hatte sich die Familie nach ihrer Rückkehr nach Europa in Sterzing in Südtirol niedergelassen. Der Ansitz Löwenegg, auf halber Höhe zwischen Brenner und Jaufenstraße gelegen, gehörte bereits in den dreißiger Jahren zum Teil Ludwig Kaas, der der im Exil in Rom lebenden Familie und vor allem den Kindern häufig Ferienaufenthalte in Löwenegg ermöglichte; in der gesunden Höhenluft hatte damals auch der Keuchhusten kuriert werden können, unter dem die Schauffschen Kinder nach dem Wechsel von der Eifel nach Italien gelitten hatten ${ }^{4}$.

Noch vor der Emigration nach Brasilien hatte Johannes Schauff den oberen Teil des Anwesens erworben, der ursprünglich Prälat Steinmann von der deutschen Botschaft im Vatikan gehört hatte und nach dessen Tod an den Bischof von Brixen verkauft worden war. Von diesem war die Wohnung zuerst gemietet und später gekauft worden; nach dem Tode von Kaas im Jahre 1952 erwarb die Familie Schauff von den Erben schließlich das gesamte Anwesen, Karin Schauff zufolge

1 Karin Schauff, Schreib mir alles, Mutter, S. 11.

2 Themen waren u.a. Buchhaltung, Finanzdispositionen, Menschenführung. Korrespondenz aus dem Jahre 1956 (IfZ, NL Schauff, Bd. 27).

3 Karin Schauff, Haus Löwenegg, Ms. in IfZ, NL Schauff, Bd. 40.

4 Karin Schauff, Das Klingelband, S. 97 ff. 
„ein altes, warmes, mütterliches Haus“, das „den vielschichtigen Traditionen Südtirols eng verhaftet" war. Vor der Ausreise nach Brasilien waren alle Einrichtungsgegenstände aus dem Haus auf dem Monte Sacro in Rom nach Löwenegg verbracht worden. Während des Krieges war das Anwesen von mehreren und unterschiedlichsten Parteien genutzt oder besser "heimgesucht“ worden, deren letzte das Haus noch 1949 besetzt hielt. Diese unerfreuliche Situation aufzulösen war neben der Option für den lateinischen Süden aber auch deshalb notwendig, weil auch das der Familie gehörende Haus in Steinfeld in der Eifel mit Evakuierten belegt war.

Die bereits in den dreißiger Jahren geknüpften Kontakte zu einigen Familien und Persönlichkeiten des öffentlichen Lebens in Südtirol wurden nach der Rückwanderung häufig zu dauerhaften Freundschaften. Dazu gehörten der Brixener Bischof Josef Gargitter ${ }^{5}$, die Sterzinger Familie Arthur und Lilly Langer ${ }^{6}$, die Familien Tschurtschenthaler und von Unterrichter in Bozen und Brixen.

Von Löwenegg aus organisierte Karin Schauff die dringende schulische Ausbildung der Kinder. Zwischen 1927 und 1942 war deren Zahl auf neun angewachsen: Eva Maria (geb. 1927), Christof (geb. 1929), Tobias (geb. 1931), Veronika (geb. 1932), Johanna (geb. 1933), die Zwillinge Nikolaus und Michael (geb. 1937), Maria Laetitia (geb. 1940) und Markus (geb. 1942). Die jüngeren - Veronika, Johanna, Michael und Nikolaus, Maria Laetitia und Markus - „mußten unverzüglich in Schulen, und so wurden sie nach Südtirol und in das dortige, uns Raum bietende Haus gebracht. Eva war bereits verheiratet, Christof blieb in Brasilien und Tobias, der nach den brasilianischen Schulen zwei Jahre im College in England war, war zu jener Zeit im Verlag Oldenbourg in München' ${ }^{7}$ Es bedeutete mehrere Jahre ungeheurer körperlicher und seelischer Anstrengungen, um die Kinder teils wieder in Europa anzusiedeln, teils sie dort Fuß fassen zu lassen. Durch die Weite und Freiheit Brasiliens und einer Farm waren sie verwöhnt; sie ordneten sich nur schwer in die Systematik und Hergebrachtheit der Alten Welt ein, so großartig landschaftlich und kulturell - Südtirol auch war ... Veronika, mit ihren praktischen Fähigkeiten als Farmerin und Weberin, ging in die landwirtschaftliche Schule nach Dietenheim, Johanna zu den Englischen Fräulein nach Brixen. In Salern machten die Zwillinge [Michael und Nikolaus] einen Schnellkurs in Italienisch und holten dann bei den Benediktinern in Meran den Stoff von drei Schuljahren in nur einem Jahr nach. Danach besuchten sie das alte Franziskanergymnasium in Bozen, wo sie das italienische Abitur in deutscher Sprache ablegten. Maria und Markus nahmen zunächst an dem deutsch-italienischen Unterricht der Bürgerschule in Sterzing teil und wurden zu Hause von deutschen Erzieherinnen unterrichtet. Dann gingen sie in Internate: Maria war zunächst ein Jahr in Kloster-

5 Josef Gargitter, geb. 1927 in Lüsen bei Bozen, wurde am 18. Mai 1952 zum Bischof von Bozen und Brixen geweiht.

6 Inhaber der Apotheke in Sterzing; der Sohn Alexander Langer, später führender Politiker der Grünen in Südtirol und Rom, war, wie der Verfasser selbst feststellen konnte, im Hause Schauff fast wie ein eigenes Kind.

7 Christof, Tobias und später auch Markus blieben als Farmer oder wurden später Farmer in Brasilien; der Jurist Michael ist leitender Angestellter (Direktor) im Verband der deutschen Milchwirtschaft, die Töchter heirateten. 
wald am Bodensee, dann Châtelaine in Neuchâtel/Schweiz, danach vier Jahre College in San Antonio/Texas, wo sie nach dem Bachelor heiratete. Markus war ein Jahr in der Stella Matutina in Feldkirch/Österreich, dann sieben Jahre in Montana, Zugerberg in der Schweiz. Alle Ferien verbrachten die gesamten Kinder in Löwenegg. In der Zeit der Umsiedlung von Südamerika nach Europa hat dieser Ansitz der Familie die größten Dienste geleistet. " 8

In die Zeit des Aufenthalts in Löwenegg fielen auch die ersten testamentarischen Überlegungen, die vor allem die Kinder und Karin Schauff betrafen'. Schauff hatte bis dahin weitgehend von den Erträgen gelebt, die auf seiner Farm im Verlauf eines Dezenniums zunehmend erfolgreich und vor allem auf der Basis des Kaffeeanbaus erwirtschaftet wurden. Dennoch wurde Karin Schauff nicht müde, wenn auch letzten Endes vergebens, für das Engagement ihres Mannes in der ICMC eine angemessene Entschädigung einzufordern. Dabei war der Ansprechpartner im Vatikan, Robert Leiber, ein langjähriger Vertrauter von Pius XII., mit dem Schauff bei der Organisation der ICMC eng zusammengearbeitet hatte ${ }^{10}$.

"Seit unserer Rückkehr aus Brasilien“, schrieb Karin Schauff anläßlich der Johannes Schauff im Sommer 1956 angetragenen ICMC-Präsidentschaft ${ }^{11}$ an Robert Leiber, „also nun schon mehr als sieben Jahren, arbeitet er im Wesentlichen für die katholische Wanderung. Das einzige, das er bisher materiell aus dieser Tätigkeit erhielt, war in den letzten beiden Jahren eine Aufwandsentschädigung, die viel zu gering war, um unsere große Familie mit neun Kindern ... zu erhalten und selbst dafür, um mit ihm zusammenleben zu können. Die Opfer, die aus der nun schon viele Jahre währenden Trennung zwischen ihm und der Familie erwachsen, sind in menschlicher und materieller Hinsicht zu groß geworden, als daß sie sich noch weiterhin rechtfertigen ließen.

Auf der anderen Seite ist es meinem Mann ein Herzensbedürfnis als einem der wesentlichen Mitbegründer der ICMC, daß die mit so viel Opfern und Mühsal aufgebaute und jetzt so blühende Organisation keinen Schaden erleidet oder gar zerfällt ...

Nun hat man meinem Mann die Präsidentschaft angetragen. Der Arbeit zuliebe würde er das auch sicher gern für einige Jahre machen, aber wegen der Familie ginge es nur dann, wenn ein Weg gefunden wird, daß wir von seinem Einkommen hier in Europa leben und die Kinder ausbilden lassen können. Dies haben wir bisher aus den Einkünften unserer brasilianischen Farm getan, die wir in Händen von

8 Karin Schauff, Haus Löwenegg, S. 3 f.

9 "Ich wünsche“ - so Johannes Schauff in einem Entwurf aus dem Jahre 1954 - „daß meine Kinder von der Erbschaft nur ihren Pflichtteil (a legitima) zu gleichen Teilen erhalten ... Ich wünsche weiterhin, daß meine Kinder bei der Erbteilung nach Möglichkeit je $70 \mathrm{Al}$ queires Kaffeeland in der vorderen Urwaldzone erhalten sollen; meine sog. freie Hälfte soll zu lebenslänglichem Nießbrauch meiner Frau Karin Schauff zufallen, die darüber frei verfügen darf.“ Im Falle von deren Tod solle deren Anteil „zu gleichen Teilen meinen Kindern zufließen“. (Dieser Entwurf und weitere Testamentsfragen betr. Schriftverkehr in: ACDP, NL Hermann Kopf, Sign. I-027-004/3).

10 Siehe S. 101.

11 Siehe oben, S. 111. 
mehr oder weniger guten Verwaltern wissen, was aber keine so große Rolle spielen würde, wenn wir hier aus der vielen Inanspruchnahme meines Mannes ein entsprechendes und geregeltes Einkommen hätten." 12

Wie schon erwähnt, mußte Karin Schauff angesichts der häufigen beruflichen Inanspruchnahme und Abwesenheit des Ehemannes mehr oder minder regelmäBig nach Brasilien reisen, um auf der die Familie ernährenden Fazenda nach dem Rechten zu sehen. Angesichts der ständigen Zeitknappheit war dabei die Benutzung des Flugzeugs geboten, was insbesondere bei Reisen innerhalb Brasiliens häufig recht abenteuerlich sein konnte. So schildert Karin Schauff eine Landung, bei der das Fahrgestell abbrach, sowie „jenen furchtbaren Flug zwischen Leben und Tod, bei dem es durch ein enges Gebirgstal ging, wo man fürchten mußte, daß die Tragflächen an den nahen Felswänden zerschellen, dann wieder fegte man über Baumkronen ... Wir flogen in die Wiesen, die Maschine drehte sich wie ein rasendes Karussell und bohrte sich mit der Schnauze in die Erde." Oft hätten die Passagiere und herbeigeholte Bauern das Flugzeug wieder flott machen müssen ${ }^{13}$.

Die Reisen innerhalb Brasiliens waren deshalb notwendig, weil die technischen Ausrüstungen der Fazenda ausgebaut und erneuert werden mußten. Gerade dieses Material bildete aber, wenn auf der Farm die Oberaufsicht fehlte, häufig ein Objekt der Begehrlichkeit für das zum Teil gewalttätige Brigantentum, das sich parallel zum Prozeß des wirtschaftlichen Aufstiegs der Siedlungen entwickelt hatte. Karin Schauff scheint - beraten durch den inzwischen auch in Brasilien als Farmer ansässigen Sohn Tobias - unter den vielfach noch abergläubischen und ängstlichen Farmarbeitern und -bediensteten häufig genug als einzige ihren kühlen Kopf bewahrt zu haben, wenn unter Umständen nur Waffen und scharfe Hunde Schutz zu bieten vermochten ${ }^{14}$. Eine alltägliche Bedrohung war auch die Plage der ausgewachsenen und aggressiven Gambas (Beutelratten), denen sich die Hausherrin manchmal nächtens in ihrem Schlafzimmer gegenübersah.

Gambas wie auch andere wilde Tiere, nicht zuletzt auch die zahlreich vorkommenden Schlangen wie die giftige und starke Chararáca, hatten jedoch seit jeher zur Fazenda gehört. Karin Schauff hat Vergleiche gezogen zu den später neu entstandenen Siedlungsgebieten, die sich durch einen häufig sterilen und amerikanisierten technischen Standard an Geräten und Fahrzeugen auszeichneten, ein Modernisierungsschub, der früher oder später aber auch die „alten“ Gebiete wie Rolândia erreichte. Der Eisschrank, so Karin Schauff, sei lange Zeit der Bach gewesen, das Verkehrsmittel der Pferdekarren. Doch sperrte sie sich nicht gegen den neuen Komfort, der der nun häufig allein residierenden Herrin der Fazenda das Leben schließlich in vielerlei Hinsicht angenehmer machen konnte.

Im europäischen Refugium in Sterzing hingegen fand Karin Schauff, nachdem die Ausbildung und Unterbringung der Kinder geregelt waren, auch die Ruhe, sich auf sich selbst zu besinnen und auch zurückzuziehen. In dieser Zeit entstanden die ersten Buchprojekte, in denen sie das Schicksal ihrer Familie rekapitulierte und ihr historisches und politisches Umfeld kritisch reflektierte. Sie knüpfte auch

12 Karin Schauff an P. Robert Leiber SJ, 28. 7. 1956 (IfZ, NL Schauff, Bd. 14).

13 Karin Schauff, Schreib mir alles, Mutter, S. 33.

14 Ebenda, S. $43 \mathrm{ff}$. 
wieder an das seinerzeit aufgegebene Medizinstudium an, beschäftigte sich mit Tiefenpsychologie und machte in der zweiten Hälfte der fünfziger Jahre in Garmisch eine sechsjährige Lehranalyse.

Die Selbstvergewisserung, der diese Lehranalyse diente, vertiefte aber auch die Möglichkeiten, Mitmenschen noch besser zu erkennen und mit ihnen zu kommunizieren. Karin Schauffs positiver Zugang zu Ludwig Kaas wurde bereits erwähnt. Eine andere Persönlichkeit, der sie begegnete und die "nach freundschaftlich-warmer Gemeinsamkeit oder Mitteilungsmöglichkeiten verlangte", war Heinrich von Brentano, ab 1955 Außenminister der Bundesrepublik Deutschland15.

Johannes und Karin Schauff waren Heinrich von Brentano bereits vor 1933 begegnet ${ }^{16}$. Nach ihrer Rückkehr nach Europa gab es ein Wiedersehen, als Johannes Schauff vor der Entscheidung stand, ob er in die deutsche Politik zurückkehren solle. Bei Schauffs politischen und diplomatischen Unternehmungen im Dienste der Bundesrepublik in den fünfziger Jahren ergab sich ein fruchtbares Zusammenspiel. Gerade Karin Schauff besaß die Fähigkeit, zu dem privatim häufig isolierten und zu Depressionen neigenden Außenminister eine tiefere menschliche und freundschaftliche Beziehung aufzubauen. Brücke dazu war wieder jenes „lateinische" Erbe, das mit der deutschen Akkulturation in unauföslicher Spannung stand, sie zugleich aber auch bereichern konnte. Die Zugehörigkeit der Familie Brentano zu Völkern germanischer und lateinischer Mentalität - so Karin Schauff - sei „eine aufschlußreiche Quelle für das Verständnis seiner eigenen vielschichtigen Persönlichkeit gewesen“.

Das konnte nur eine Persönlichkeit voll ermessen, die wie sie selbst Jahrzehnte als Deutsche unter Lateinern gelebt und dort Kinder erzogen hatte. Aus solchen Quellen hatte nach Einschätzung Karin Schauffs auch Brentano immer wieder die Kunst genährt, gegensätzliche Eigenschaften von Menschen und Gruppen, die einander an sich widersprechenden Problemkomplexen gegenüberstanden, zu achten und in der Waage halten zu können. Daraus erwuchs die Schätzung der eigenen - und fremder - Freiheit als hohes Gut sowie die Fähigkeit zu wahrer Toleranz den Meinungen und Handlungen anderer gegenüber. ${ }^{17}$

Brentano war häufiger Gast in der Wohnung in der Via Gregoriana in Rom, die Karin und Johannes Schauff im Vorfeld der Vorbereitung des Zweiten Vatikanischen Konzils bezogen hatten ${ }^{18}$. In Rom, Karin Schauff zufolge die „mütterlichste aller Städte, brauchte er [Brentano] sich überhaupt keinen $\mathrm{Zwang}$ anzutun, konnte er so sein, wie er vom Grunde seines Wesens wirklich war. Dort fühlte er sich zu Hause und befriedet - in Frieden auch mit sich selbst." 19 Als Brentano im Dezember 1963 schwer erkrankte - er starb ein Jahr darauf am 14. November 1964 - betreute Karin Schauff den Freund in seinem Haus in Darmstadt bis zu seinem Tode.

15 Karin Schauff, Brentano, S. 49.

16 Mitteilung Karin Schauffs an den Verfasser.

17 Karin Schauff, Brentano, S. 48 f.

18 Siehe unten, Kap. X, S. 165.

19 Karin Schauff, Brentano, S. 51. 